This is an electronic reprint of the original article. This reprint may differ from the original in pagination and typographic detail.

Author(s): Joffre, Thomas; Miettinen, Arttu; Berthold, Fredrik; Gamstedt, E. Kristofer

Title: X-ray micro-computed tomography investigation of fibre length degradation during the processing steps of short-fibre composites

Year: $\quad 2014$

Version:

Please cite the original version:

Joffre, T., Miettinen, A., Berthold, F., \& Gamstedt, E. K. (2014). X-ray micro-computed tomography investigation of fibre length degradation during the processing steps of short-fibre composites. Composites science and technology, 105(December), 127133. https://doi.org/10.1016/j.compscitech.2014.10.011

All material supplied via JYX is protected by copyright and other intellectual property rights, and duplication or sale of all or part of any of the repository collections is not permitted, except that material may be duplicated by you for your research use or educational purposes in electronic or print form. You must obtain permission for any other use. Electronic or print copies may not be offered, whether for sale or otherwise to anyone who is not an authorised user. 


\title{
X-ray micro-computed tomography investigation of fibre length degradation during the processing steps of short-fibre composites
}

\author{
Thomas Joffre $^{\mathrm{a}}$, Arttu Miettinen ${ }^{\mathrm{b}}$, Fredrik Berthold ${ }^{\mathrm{c}}$, E. Kristofer Gamstedt ${ }^{\mathrm{a}, *}$ \\ ${ }^{a}$ Division of Applied Mechanics, Department of Engineering Sciences, Uppsala University, Box 534, \\ SE-75121 Uppsala, Sweden \\ ${ }^{b}$ Department of Physics, University of Jyväskylä, P.O. Box 35 (YFL) FI-40014 Jyväskylä, Finland \\ ${ }^{c}$ Innventia AB, Packaging Solutions, Box 5604, SE-114 86 Stockholm, Sweden
}

\begin{abstract}
The mechanical properties of composites in the fibre direction are mainly attributed to the fibre slenderness, or aspect ratio. A trade-off between performance and processability is usually required, and dependent on the intended application. If the fibre length could be retained or not severely degraded during various processing steps towards the injection-moulded component, a stiffer and stronger composite product could be obtained. The processing steps for injection moulded wood-fibre composites here include: Pulping, commingling, extrusion, pelletizing, and injection moulding. To tune the processing parameters systematically for retained fibre length, it would be useful to investigate the degradation of the original fibre length distribution throughout the processing chain. The fibre length degradation has been monitored by X-ray micro-computed tomography through the processing steps in wood pulpfibre reinforced polylactide. A significant fibre-length degradation was found, in particular, the extrusion step was found to result in a drastic fibre length reduction.
\end{abstract}

Keywords: A. Polymer-matrix composites (PMCs), B. Microstructures, E. Injection moulding, X-ray tomography

\section{Introduction}

Wood pulp fibres are readily available as a renewable reinforcement for composite materials. The fibre length (individual tracheid cell length) is about 1-4 $\mathrm{mm}$ for softwood fibres

\footnotetext{
*Corresponding author. Tel. +4618-471 3026

Email address: kristofer.gamstedt@angstrom.uu.se (E. Kristofer Gamstedt)
} 
(see e.g. [1 3]). The wood fibres are still sufficiently short to be used in melt processing of thermoplastic-matrix composites, and result in considerable less wear of tools and dies than e.g. short glass fibres. With a diameter of about $25 \mu \mathrm{m}$, the relatively high aspect ratio makes these fibres interesting from a reinforcement perspective compared to wood flour, where the reinforcing particles have much lower aspect ratio [4]. However, during the manufacturing processes, the fibres are worn down to very small aspect ratios; sometimes even to particle size. With a particle-like geometry the wood fibres are acting as a filler rather than as a reinforcement. Strength could even be lower than that of the neat matrix if the cellulose fibre segments are too short [5]. For sufficiently long fibre segments, i.e. a couple of times longer than the ineffective length of stress-build up at the end of an oriented embedded fibre, the desired reinforcement can be achieved. In a complicated melt processing procedure, there are several steps that individually contribute to the degradation of the initial fibre length, and hence also to the inferior mechanical properties, as compared e.g. with resin infusion methods, where the fibres in the fibre mat are basically intact after impregnation and curing. In this study, we focus on the manufacturing of injection-moulded wood pulp-fibre reinforced polylactide (PLA), i.e. an entirely renewable and biodegradable composite material. The processing steps for these injection-moulded wood-fibre composites are pulping, commingling, extrusion, pelletizing and injection moulding. The fibre length distribution is quantified step-by-step along the processing line. If the step which results in the most severe fibre degradation could be identified, measures could be taken to mitigate and treat the fibres more gently at this step to retain some of its slenderness. Subsequently, this procedure could be repeated to identify a stage further downstream that is liable for the most serious fibre degradation in the updated processing chain. Of course, there is a balance between processability and mechanical performance, and some fibre length reduction must be acceptable in order to keep the high production rate of injection-moulded composite products leading to low manufacturing costs. The quantification of fibre length distributions in composites has traditionally been done by chemically dissolving the matrix and studying a dilute fibre suspension by optical microscopy, see e.g. [6]. The fibre length distribution can then be quantified either manually by measuring the fibre lengths one by one, or by using two-dimensional image analysis. The dissolution and optical microscopy 
procedures are direct, but rather time consuming. Fibre lengths in water suspensions could be more swiftly characterized by on-line systems where the projected dimensions of individual fibres are measured digitally as they flow in a controlled manner, one by one, through a capillary tube. The Innventia FiberMaster [7, 8] and Kajaani Fiberlab [9] are examples of such equipment. X-ray microcomputed tomography $(\mathrm{X} \mu \mathrm{CT})$ is a non-destructive method to assess the three-dimensional structure of a sample from two-dimensional X-ray projection images. After computational processing of the projection images, a three-dimensional array of X-ray attenuation coefficients is obtained. For many materials, especially non-diffractive ones, the attenuation coefficient correlates with local density of the material. Thus, the method essentially produces a density map of the sample. Recent development of $\mathrm{X} \mu \mathrm{CT}$ methods has conveyed the composite research community with a non-destructive technique capable of characterizing the geometry of fibres inside the composite material sample. With the aid of 3D image analysis, it is possible to quantify fibre length, diameter and orientation distributions from the reconstructed 3D images, as shown by Luengo Hendriks [10], Miettinen et al. [11, 12] and Joffre et al. [13]. Some interesting previous studies on this general topic include the work by Alemdar et al.[14], who showed that $\mathrm{X \mu CT}$ can be used to quantify the dimensions of wood and flax fibre in polypropylene matrix. In that case the Image-Pro Plus software was used to identify the Weibull parameters of the size distributions of the fibres.

\section{Experimental procedures}

\subsection{Materials}

The natural fibres used for making the composites were bleached sulphite pulp fibres extracted from Norway spruce (Picea Abies) delivered from Innventia AB. The matrix material was polylactic acid (PLA). PLA is thermoplastic aliphatic polyester manufactured from starchrich renewable resources such as maize, sugar beets or wheat. For a biopolymer, PLA has decent physical and mechanical properties, making it a candidate for substitution of certain petrochemical thermoplastics. Due to the good adhesion to cellulose fibres, it has been proven to be suitable to use PLA as matrix material in pulp-fibre composites [15, 16]. The PLA fibres used here were PLA 01 quality delivered from Unitika (1.7 dtex, $1 \mathrm{~mm}$ length). The polymer 
fibres were first run through a pulp screen (Wennbergsil) and dried at room temperature in order to facilitate filament release.

\subsection{Manufacturing}

Commingled wood fibre/PLA fibre mats were manufactured using a laboratory-scale dynamicsheet former. The polymer and wood fibres were evenly distributed, limiting the fibres to aggregate into larger bundles, which has been observed to be the case when PLA pellets and pulp fibres are introduced directly in the extruder [17]. The extrusion experiments were run on a $40 \mathrm{~mm}$ co-rotating twin screw extruder, Werner \& Pfleiderer ZSK40. The material was run through the extruder either once or twice, as shown in Fig. 1 Multiple extrusion runs will reduce porosity and improve compounding and surface finish, but most likely reduce the average fibre length and thus impair mechanical properties. The extrudates and pellets obtained after two extrusions were less rough and thus easier to process (see Fig. 1). The extrudates were cooled in water and led to a chopper to produce pellets. After pelletizing the material was dried in an oven at $50^{\circ} \mathrm{C}$ for several hours. Extrudates were also broken into smaller pieces using a hammer mill. The weight fraction of wood fibres, $w_{f}$, was kept constant at $30 \%$. The weight fraction is more convenient to measure during the manufacturing, whereas the volume fraction, $V_{f}$, is used as input in micromechanical models and can be tracked by binarization of $\mathrm{X} \mu \mathrm{CT}$ images. The volume fraction can be estimated from measurable quantities, using the relation

$$
V_{f}=\frac{w_{f} \rho_{f}}{w_{f} \rho_{f}+w_{m} \rho_{m}}
$$

where $\rho_{f}$ is the density of the fibres, $1.5 \mathrm{~g} / \mathrm{cm}^{3}$ [18], and $\rho_{m}$ is the density of the PLA, 1.25 $\mathrm{g} / \mathrm{cm}^{3}$ [19]. Thus the volume fraction of fibre introduced here is $26.3 \%$. After drying the pellets were fed into the injection moulding machine equipped with a multiple die containing mould profiles for dogbone shaped specimens. At each step of the process, samples have been taken off for $\mathrm{X} \mu \mathrm{CT}$ analyses.

\subsection{X-ray computed microtomography}

To prepare the composite samples for the $\mathrm{X} \mu \mathrm{CT}$ imaging, a rotary tool was used to carve the sample into a cylinder with radius of approximately $1 \mathrm{~mm}$. The cylinder was then glued to the 
(a)



(b)



Figure 1: Extrudates as they leave the extruder, after (a) one pass, and (b) after two passes through the extruder. Note that the apparent quality of the extrudates is improved after a second pass. The improved processability is at the cost of shortened fibre length.

top of a carbon fibre tube that served as a sample holder. To scan pulp fibres and commingled fibres, no specific sample preparation was needed; a bundle of fibres was simply glued on top of a glass tube acting as a sample holder. The X $\mu \mathrm{CT}$ imaging was done using Xradia MicroCT-400 tomograph. For composite materials a resolution of approximately $1 \mu \mathrm{m}$ was chosen, resulting in field of view of approximately $1 \mathrm{~mm}^{3}$. Pulp and commingled fibres were longer, so field of view of approximately $8 \mathrm{~mm}^{3}$ was used with $2 \mu \mathrm{m}$ resolution. A typical cross-sectional slice through a sample is visualized in Fig. 2a and some three-dimensional visualizations are drawn in Fig. 4. For measuring the void content, it was necessary to use large samples to have sufficiently large and representative volumes. In this case, entire pellets or machined pieces of the injection-moulded sample were scanned using a Skyscan 1172 tomograph. The larger samples resulted in lower resolution, about $3 \mu \mathrm{m}$, which is still sufficiently high to detect the vast majority of the typical voids.

\subsection{Image analysis}

To separate the fibres from the matrix in the three-dimensional images, a variance weighted mean filter was first applied in order to attenuate imaging noise. The images were then thresholded. Objects having volume smaller than 5000 voxels, corresponding to a sphere whose radius is less than 10 pixels, were considered as noise and removed. A typical result of these operations is presented in Fig. 2. The determination of fibre length and cross-sectional 
(a)



Figure 2: Visualisation of a slice of the three dimensional images, (a) just after reconstruction, and (b) after segmentation. The width of the enlarged image is approximately $1 \mathrm{~mm}$.

area was done as in [11, 12]. The method is based on estimation of the local orientation of the fibres that facilitates extraction of cross-sectional slices. The cross-sectional area of fibres is determined from the slices. The fibre length is estimated using the constrained path-opening method as in [10] and [11, whose results are then correlated to the cross-sectional properties. The fibres are sampled statistically such that the results are expressed as length and crosssectional area distributions of fibre volume. A brief description of this method is given in Appendix.

\section{Results and discussion}

Softwood tracheids from species like Norwegian spruce and Scots pine have a native length of up to $4 \mathrm{~mm}$ [1]. These fibres are inevitably shortened during each of the following processing steps towards the wood-fibre composite. The wood is chipped, pulp cooked, commingled with the thermoplastic fibres, extruded once or twice, pelletized, and finally injection moulded to form a fibre-composite component. In Fig. 3, a three-dimensional reconstruction of the microstructure of the commingled material is found. During the manufacturing process, the slender green fibres may thus even be degraded in length to the point of being particles rather than long reinforcing fibres. The fibre degradation which occurs during the manufacturing 




Figure 3: Visualisation of $\mathrm{X} \mu \mathrm{CT}$ images of the commingled wood and PLA fibres. The width of the sample is approximately $1 \mathrm{~mm}$.

process is shown in Fig. 4, where three-dimensional visualisations of (a) wood pulp fibres and (b) fibres within a composite specimen are compared.

With the image analysis methods described in the previous section, it is possible to quantify the distribution functions of the fibre length and cross-sectional area at each stage of the manufacturing process. The results are presented in Fig. 5. From the fibre length distribution, the average length of a fibre has been calculated for each sample (Fig. 6). The number average will depend on the chosen threshold level of the fibre length. Also weighted arithmetic averages of higher orders, such as the mass average, will be affected by the threshold level, although to a lesser degree. The number average was chosen here, since its interpretation is straightforward, and the threshold is set to a fibre aspect ratio about unity, at which the fibres cannot be considered as a reinforcement but only a filler.

It can be noticed in Fig. 5 that the fibre length distribution of the intact fibres, i.e. the separated pulp fibres, is already significantly shifted to shorter segments in comparison to what is expected for mildly pulped fibres [20]. Although the fibres have become shorter in the pulping process compared with the green native state, an average length of $600 \mu \mathrm{m}$ means that the fibres can be considered slender from a reinforcing point of view, with an aspect ratio of more than 20. The commingling process was gentle to the fibre, and did not lead to any significant 
(a)



(b)



Figure 4: Visualisation of $\mathrm{X \mu CT}$ images of (a) a dry and unimpregnated bundle of pulp fibres, and (b) pulp fibres in a composite sample. The width of the sample is approximately $1 \mathrm{~mm}$ in both figures.

(a)



(b)



Figure 5: Measured density distributions after each processing step for (a) the fibre length, and (b) the cross sectional area. 




Figure 6: Illustration of the fibre length degradation through the processing chain.

fibre length degradation. The most important degradation occurred during the extrusion and injection moulding steps. The average fibre length was found to be $300 \mu \mathrm{m}$ for the material extruded once and $250 \mu \mathrm{m}$ for the material extruded twice. The effect of the hammer mill on the fibre length distribution is assumed negligible since the pieces were about one order of magnitude longer compared to the typical length of a fibre after extrusion. This length degradation is further worsened after the injection moulding step. The average fibre length of the injection moulded specimen was $160 \mu \mathrm{m}$. Whether the material has been extruded once or twice does not have any influence on the fibre length in the final injection-moulded samples. The twice-extruded materials were, however, much better compounded. The cross-sectional area measured at each processing step shows a similar decreasing trend as the fibre length, but the relative decrease is smaller. There is an increasing amount of debris and small fibre fragments, but the cross-sectional area of the larger fibres remain principally intact, represented by the upper tail in Fig. 5 b.

The obtained values of average length are lower but in the same order of magnitude, and show similar trends as those obtained by optical microscopy by Nyström et al. [21] for chemothermomechanical pulps in injection-moulded polypropylene-matrix composites. Our lower values can be attributed to the resulting embrittlement in the sulphite cooking process, as compared to kraft or thermomechanical pulps [22]. Oksman et al. [23] investigated the fibre length degradation and concomitant tensile properties of injection-moulded composites based on a number of different types of natural fibres in polypropylene matrix. The fibre fragmentation 
was dependent on the species of natural fibres. Some natural fibres are more brittle and likely to break during processes than others. Nyström [24] have reported a rather comprehensive study in her thesis on the effects of the processing parameters on the strength of natural fibre composites. She found that the extrusion speed at the compounding stage, and the injection speed and temperature at the injection-moulding stage had the highest impact on the tensile properties, which is controlled by the fibre aspect ratio for natural-fibre composites with rather weak interfacial coupling, such as the case of polypropylene matrix systems. It should be emphasized that the density functions presented in Fig. 5 have been obtained by only considering objects with a volume larger than the threshold value of 5000 voxels (i.e. approximately 5000 $\mu^{3}$ ). Smaller objects are considered as debris and do not essentially contribute to the reinforcement and strength of the composite, since the corresponding aspect ratio is close to 1 . When fibres are cut, some parts might have such a small size that they were considered as noise during the image analysis treatment. From fraction of fibres introduced in the process obtained by Eq. 1, and the volume of fibre visible in the segmented three dimensional images, it is possible to calculate the percentage of fibres having a size less than $5000 \mathrm{\mu m}^{3}$, roughly corresponding to a fibre length of $20 \mu \mathrm{m}$ (considering the average cross sectional area measured). The amount of debris or non-reinforcing particles is shown in Fig. 7a. The trend is that the amount of small fragments is increasing throughout the processing chain. Although there is an increasing trend, it can be noted that there is an unexpected reduction in fragment volume fraction of particles from the composite extruded once to that extruded twice, which can only be explained to incertitude in measurements or agglomeration of particles which can occurred in the twice extruded material [17].

The amount of fragments is considerable, up to $48 \%$ by volume of introduced wood in the most degraded samples. Although the higher tail with long fibres contributes significantly more to the strength of composites that small fragments, it is an inefficient use of raw material if almost half of the fibres ends up as a non-reinforcing particulate filler. It is therefore motivated to develop gentler processing techniques which would make more efficient use of initially slender fibres. The volume fractions of voids are presented for each processing step in Fig. 7b and three-dimensional visualizations are drawn in Fig. 8. It can be noted that the void decreases 



Figure 7: Effect of the processing chain on (a) the relative amount of wood fibres with a volume below the threshold value of $5000 \mathrm{\mu m}^{3}$, and(b) the volume fraction of voids, after extrusion and injection moulding.

for every extrusion step and after injection moulding. By ocular inspection, the samples appear to have become more homogenous after each manufacturing step. At the price of decreased bre length, the porosity can be signicantly reduced. A compromise between a low void content and high bre aspect ratio has to be found in order to optimise the bre composite. It should also be mentioned that increasing the number of processing step might also induce thermal degradation of PLA.

\section{Conclusion}

It was possible to use X-ray microtomography to assess the microstructure of wood fibre composite, and thus quantify the fibre length distribution and the void content through the compounding and injection moulding process. The fibres are highly damaged during the manufacturing process of wood fibre composite, and their average length is significantly reduced. The first commingling process step used to mix pulp and thermoplastic fibres is gentle to the fibres without any serious fibre degradation, whereas the extrusion and injection moulding steps have been identified as responsible for the most severe reduction of the fibre length, meaning reduced mechanical properties of the final composite material. An opposite trend was found for the void content. The porosity decreased monotonically for each processing step, resulting 
(a)

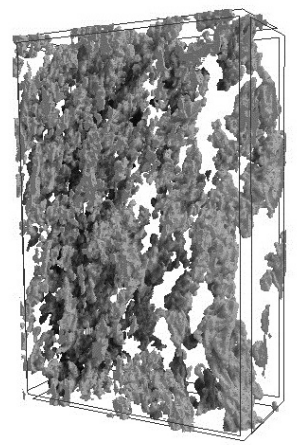

(b)

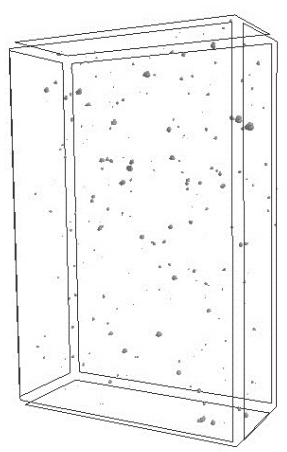

Figure 8: Three dimensional visualization of the void content (a) in a pellet (22\%), (b) after extrusion and injection moulding $(2 \%)$. The width of the each image is approximately $1 \mathrm{~mm}$.

in a more homogenous composite. A trade-off between porosity and fibre length must be made, depending on if surface finish or load-carrying capacity is the more important property in the intended application. Essentially, it can be concluded that X-ray micro-computed tomography is a suitable and expedient tool to quantify the microstructural parameters of short-fibre composites in a reproducible and user-independent manner compared with optical microscopy of fibres dissolved from the matrix. Tuning of processing parameters can be facilitated by investigating the effects on the key microstructural parameters.

\section{Acknowledgements}

The computations were performed on resources provided by the Swedish National Infrastructure for Computing (SNIC), through Uppsala Multidisciplinary Center for Advanced Computational Science (UPPMAX). Financial support was provided by Formas and the WoodWisdomNET project WoodFibre3D.

\section{Appendix. Estimation of fibre length and cross-sectional area}

The local orientation of fibres in a three-dimensional binary image $I$ can be estimated by the eigenvector corresponding to the smallest eigenvalue of the structure tensor $S_{i j}$ [25], defined by

$$
S_{i j}=G\left(\sigma_{t}\right) *\left(I_{i} I_{j}\right)
$$




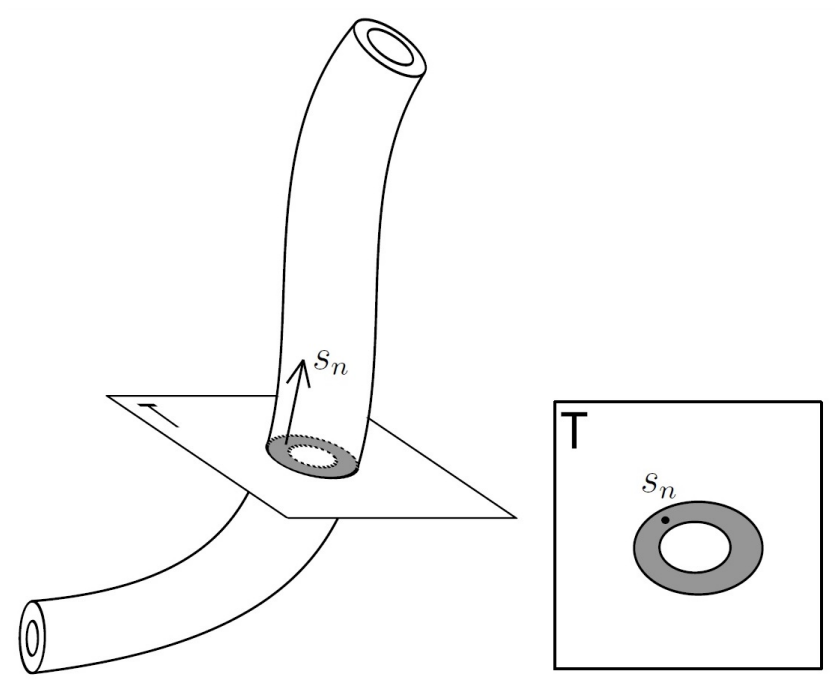

Figure 9: Slicing of the fibre, where the slice $\mathrm{T}$ is taken orthogonal to the fibre orientation vector $s_{n}$.

where $G(\sigma)$ is a Gaussian function with zero mean $\sigma$ variance in all directions. The partial derivatives $I_{i}$ can be approximated by

$$
I_{i}(\mathbf{p})=\frac{\partial I(\mathbf{p})}{\partial x_{i}} \approx\left(\frac{\partial G\left(\sigma_{s}\right)}{\partial x_{i}} * I\right)(\mathbf{p}) .
$$

The image $I$ is sampled uniformly at random locations $\mathbf{x}_{n}$ inside fibre wall. At each location, a slice around the point $\mathbf{x}_{n}$ is extracted such that the normal of the slice coincides with the local fibre orientation, as shown in Fig. 9. The cross-section of the fibre at $\mathbf{x}_{n}$ is visible in the slice and its area can then be determined directly.

The length of the fibres is estimated using the constrained path transform algorithm [10, 11], whose result $T(\mathbf{x})$ gives the length of the longest approximately straight path going through $\mathbf{x}$ and staying inside fibre wall. The length of the fibre at $\mathbf{x}_{n}$ is then taken to be the mode of the values of $T$ over the $n$ :th cross-sectional slice.

\section{References}

[1] S. L. Bardage, Three-dimensional modeling and visualization of whole norway spruce latewood tracheids, Wood and Fiber Science 33 (4) (2001) 627-638.

[2] A. Bledzki, M. Letman, A. Viksne, L. Rence, A comparison of compounding processes and wood type for wood fibrepp composites, Composites Part A 36 (6) (2005) 789-797. 
[3] J. Brändström, Micro-and ultrastructural aspects of norway spruce tracheids: a review, IAWA Journal 22 (4) (2001) 333-354.

[4] N. M. Stark, R. E. Rowlands, Effects of wood fiber characteristics on mechanical properties of wood/polypropylene composites, Wood and Fiber Science 35 (2) (2003) 167-174.

[5] P. Balasuriya, L. Ye, Y.-W. Mai, Mechanical properties of wood flake polyethylene composites. part I: effects of processing methods and matrix melt flow behaviour, Composites Part A 32 (5) (2001) 619-629.

[6] R. Mittal, V. Gupta, P. Sharma, The effect of fibre orientation on the interfacial shear stress in short fibre-reinforced polypropylene, Journal of Materials Science 22 (6) (1987) 1949-1955.

[7] B. N. Nguyen, S. K. Bapanapalli, J. D. Holbery, M. T. Smith, V. Kunc, B. J. Frame, J. H. Phelps, C. L. Tucker, Fiber length and orientation in long-fiber injection-molded thermoplastics part I: Modeling of microstructure and elastic properties, Journal of Composite Materials 42 (10) (2008) 1003-1029.

[8] H. Karlsson, P. Fransson, STFI fibermaster gives papermakers new muscle-new knowledge concerning fiber shape can be the key to paper of the future, Svensk PapperstidningNordisk Cellulosa 97 (10) (1994) 26-28.

[9] Y. Cöpür, H. Makkonen, Precision and accuracy studies with Kajaani fiber length analyzers, J Appl Sci 7 (2007) 1043-1047.

[10] C. L. Luengo Hendriks, Constrained and dimensionality-independent path openings, Image Processing, IEEE Transactions on 19 (6) (2010) 1587-1595.

[11] A. Miettinen, C. Luengo Hendriks, G. Chinga-Carrasco, E. Gamstedt, M. Kataja, A non-destructive x-ray microtomography approach for measuring fibre length in short-fibre composites, Composites Science and Technology 72 (13) (2012) 1901-1908.

[12] A. Miettinen, R. Joffe, B. Madsen, K. Nättinen, K. Nättinen, Quantitative analysis of length-diameter distribution and cross-sectional properties of fibers from three- 
dimensional tomographic images, in: 34th Risø International Symposium on Materials Science, 2013, pp. 303-311.

[13] T. Joffre, E. L. Wernersson, A. Miettinen, C. L. Luengo Hendriks, E. K. Gamstedt, Swelling of cellulose fibres in composite materials: constraint effects of the surrounding matrix, Composites Science and Technology 74 (2013) 52-59.

[14] A. Alemdar, H. Zhang, M. Sain, G. Cescutti, J. Mssig, Determination of fiber size distributions of injection moulded polypropylene/natural fibers using x-ray microtomography, Advanced Engineering Materials 10 (1-2) (2008) 126-130.

[15] M. Huda, L. Drzal, M. Misra, A. Mohanty, Wood fiber reinforced poly (lactic acid) composites: Evaluation of the physicomechanical and morphological properties, Journal of Applied Polymer Science 102 (5) (2006) 4856-4869.

[16] M. Huda, L. Drzal, A. Mohanty, M. Misra, Chopped glass and recycled newspaper as reinforcement fibers in injection molded poly (lactic acid)(PLA) composites: A comparative study, Composites Science and Technology 66 (11-12) (2006) 1813-1824.

[17] T. Joffre, A. Miettinen, E. L. Wernersson, P. Isaksson, E. K. Gamstedt, Effects of defects on the tensile strength of short-fibre composite materials, Mechanics of Materials 75 (2014) $125-134$.

[18] R. Kellogg, F. Wangaard, Variation in the cell-wall density of wood, Wood and Fiber Science 1 (3) (1969) 180-204.

[19] M. Huda, L. Drzal, A. Mohanty, M. Misra, Effect of fiber surface-treatments on the properties of laminated biocomposites from poly (lactic acid)(PLA) and kenaf fibers, Composites Science and Technology 68 (2) (2008) 424-432.

[20] J. Ai, U. Tschirner, Fiber length and pulping characteristics of switchgrass, alfalfa stems, hybrid poplar and willow biomasses, Bioresource Technology 101 (1) (2010) 215-221. 
[21] B. Nyström, R. Joffe, R. Långström, Microstructure and strength of injection molded natural fiber composites, Journal of reinforced plastics and composites 26 (6) (2007) 579599.

[22] A. Johansson, O. Aaltonen, P. Ylinen, Organosolv pulping-methods and pulp properties, Biomass 13 (1) (1987) 45-65.

[23] K. Oksman, A. P. Mathew, R. Långström, B. Nyström, K. Joseph, The influence of fibre microstructure on fibre breakage and mechanical properties of natural fibre reinforced polypropylene, Composites Science and Technology 69 (11) (2009) 1847-1853.

[24] B. Nyström, Licenciate thesis; department of applied physics and mechanical engineering; natural fiber composites: optimization of microstructure and processing parameters, Thesis, Luleå University of Technology (2007).

[25] B. Jähne, Practical Handbook on Image Processing for Scientific and Technical Applications, CRC Press, 2004. 